\title{
Offset-free tracking MPC: A tutorial review and comparison of different formulations
}

\author{
Gabriele Pannocchia
}

\begin{abstract}
Offset-free Model Predictive Control formulations refer to a class of algorithms that are able to achieve output tracking of reference signals despite the presence of plant/model mismatch or unmeasured nonzero mean disturbances. The general approach is to augment the nominal system with disturbances, i.e. to build a disturbance model, and to estimate the state and disturbance from output measurements. Some alternatives are available, which are based on a non augmented system with state disturbance observer, or on velocity form representations of the system to be controlled. In this paper, we review the disturbance model approach and two different approaches in a coherent framework. Then, differently from what is reported in the literature, we show that the two alternative formulations are indeed particular cases of the general disturbance model approach.
\end{abstract}

\section{INTRODUCTION}

Model Predictive Control (MPC) solves, at each decision time, an optimal control problem in which control and state trajectories are related by a nominal model of the controlled process. The cost function typically takes into account the future (i.e. predicted) output tracking error and the input variation (or deviation from a target). The first input of the optimal control sequence is injected into the plant, and the overall algorithm is repeated again the next decision time given the new current state. If the actual process and the nominal model perfectly match, under mild assumptions this feedback control scheme can be shown to be stabilizing, and the output tracks any reachable target without offset.

Whenever permanent, nonzero mean, disturbances are present and/or there is mismatch between the MPC model and the actual plant, a more elaborate strategy is required to achieve offset-free tracking of the output. The earliest ones as Dynamic Matrix Control (see e.g. [1, Sec. 5.2]), applicable only to open-loop stable systems, were based on the use of step-response models for prediction of the nominal output response to which a constant output correction term is added. This correction term is defined as the difference between the actual and the nominal output, and represents a particular form of disturbance model, namely the output disturbance model. State-space MPC formulations based on more general disturbance models and observers have been proposed and discussed more recently [2]-[8]. In these formulations, the nominal system is augmented with integrating states (called disturbances), which are estimated from the output measurements by means of an observer, designed for the augmented system. In the case of measurable state, Tatjewski [9] discussed a state disturbance formulation that does

G. Pannocchia is with Department of Civil and Industrial Engineering, Univ. of Pisa, Italy (gabriele.pannocchiaeunipi.it). not require state augmentation. This method was recently extended to the case of output feedback [10] by adding a state disturbance estimator and an appropriate output bias term. Alternative state-space formulations are based on socalled velocity form representations of the system, in which the input and (possibly) the state are replaced by their rateof-change (see e.g. [11]-[14] and references therein). Offsetfree control is achieved by this approach without the need of defining and estimating disturbances.

It is arguable that every method has some merits and some drawbacks. This paper has three main objectives.

1) Review three different formulations within a unified framework in order to establish more easily assumptions and requirements of each method.

2) Show that the state disturbance observer method [10] and the velocity form approach are both particular cases of the general disturbance model approach.

3) Transfer the ease of tuning of the state disturbance observer method [10] to the other methods.

Notation: $\mathbb{C}, \mathbb{R}$ and $\mathbb{Z}$ denote the fields of complex, real, and integer numbers, respectively. Given $x: \mathbb{Z} \rightarrow \mathbb{R}^{n}$, we define $\delta x: \mathbb{Z} \rightarrow \mathbb{R}^{n}$ as $\delta x(k):=x(k)-x(k-1)$. A real square matrix is said to be Hurwitz if all its eigenvalues lie in the interior of the unit circle. Given $x \in \mathbb{R}^{n}$ and a positive semi-definite matrix $Q \in \mathbb{R}^{n \times n}$, we denote $\|x\|_{Q}^{2}:=x^{\prime} Q x$.

\section{PRELIMINARIES}

\section{A. Actual system, nominal model and main assumptions}

We consider linear, discrete-time, time-invariant systems:

$$
\begin{aligned}
x^{+} & =A x+B u+w \\
y & =C x+v
\end{aligned}
$$

in which $x \in \mathbb{R}^{n}, u \in \mathbb{R}^{m}, y \in \mathbb{R}^{p}$ are the system state, input and output at a given time, respectively, $x^{+}$is the successor state, $w \in \mathbb{R}^{n}$ is the state disturbance, $v \in \mathbb{R}^{p}$ is the output disturbance. Disturbances are unknown but satisfy: $w(k) \in$ $\mathbb{W} \subset \mathbb{R}^{n}$ and $v(k) \in \mathbb{V} \subset \mathbb{R}^{p}$ for all $k \in \mathbb{Z}$.

Assumption 1: The plant output $y$ is measured at each time $k \in \mathbb{Z}$. The pair $(A, B)$ is stabilizable, the pair $(C, A)$ is detectable, and the following condition holds true:

$$
\operatorname{rank}\left[\begin{array}{cc}
A-I & B \\
C & 0
\end{array}\right]=n+p
$$

Remark 2: Condition (2) is necessary for the output $y$ to track an arbitrary target $\bar{y}$, and implies that $p \leq m$.

The nominal system dynamics is given by:

$$
\begin{aligned}
\hat{x}^{+} & =A \hat{x}+B u \\
\hat{y} & =C \hat{x}
\end{aligned}
$$


The nominal state needs to be estimated at each sampling time given the output measurement. Among the various available methods, without loss of generality, we restrict our attention to the so-called current observer in which, at each time $k$, the current state prediction computed at time $k-1$ is updated using the measured output $y(k)$ as follows:

$$
\hat{x}(k)=\underbrace{A \hat{x}(k-1)+B u(k-1)}_{\text {nominal prediction }}+\underbrace{K\left(y(k)-\hat{y}^{*}(k)\right)}_{\text {correction }}
$$

in which

$$
\hat{y}^{*}(k):=C(A \hat{x}(k-1)+B u(k-1))
$$

is the predicted output, and $K \in \mathbb{R}^{n \times p}$ is the observer gain. By combining (1), (4) and (5), we can obtain the dynamics of the state estimate error, defined as $\varepsilon:=x-\hat{x}$, as follows:

$$
\begin{aligned}
\varepsilon(k)= & A x(k-1)+B u(k-1)+w(k-1) \\
& -\left[A \hat{x}(k-1)+B u(k-1)+K\left(y(k)-\hat{y}^{*}(k)\right)\right] \\
& =(A-K C A) \varepsilon(k-1)+(I-K C) w(k-1)+K v(k)
\end{aligned}
$$

\section{B. Optimal control problem formulation}

Let $\tilde{x}$ be the current state and $\tilde{u}$ the previously applied input. Let $\mathbf{x}=\left[\begin{array}{llll}x(0) & x(1) & \cdots & x(N)\end{array}\right]$, and $\mathbf{u}=$ $\left[\begin{array}{llll}u(0) & u(1) & \cdots & u(N-1)\end{array}\right]$ be, respectively, a state sequence and an input sequence. The Finite Horizon Optimal Control Problem (FHOCP) that is solved at each time, denoted by $\mathbb{P}\left(\tilde{x}, \tilde{u}, \tilde{d}_{x}, \tilde{d}_{y}\right)$, is the following:

$$
\begin{gathered}
\min _{\mathbf{x}, \mathbf{u}} \sum_{i=0}^{N-1} \ell(e(i), \delta u(i))+V_{f}(x(N), u(N-1)) \\
x(0)=\tilde{x}, \quad u(-1)=\tilde{u} \\
x(i+1)=A x(i)+B u(i)+\tilde{d}_{x} \\
u(i) \in \mathbb{U}, \quad \delta u(i) \in \delta \mathbb{U} \\
y(i):=C x(i)+\tilde{d}_{y} \in \mathbb{Y}
\end{gathered}
$$

in which $\delta u(i):=u(i)-u(i-1)$ is the control increment, $e(i):=\hat{y}(i)-\bar{y}=C x(i)+\tilde{d}_{y}-\bar{y}$ is the predicted tracking error, and the cost function $\ell(\cdot)$ is given by

$$
\ell(e, \delta u):=\|e\|_{Q}^{2}+\|\delta u\|_{R}^{2}
$$

where $\bar{y}$ represents the desired (achievable) output target. The matrices $Q$ and $R$ are assumed to be positive definite. The sets $\mathbb{U}, \delta \mathbb{U}, \mathbb{Y}$ are assumed to be polyhedral. The vectors $\tilde{d}_{x} \in \mathbb{R}^{n}$ and $\tilde{d}_{y} \in \mathbb{R}^{p}$ represent the predicted state and output disturbances (cfr. (6c) and (6e)), and they are precisely defined for each offset-free method later on. $V_{f}(\cdot)$ is the terminal cost, which may vary depending on the specific MPC formulation. In order for $\mathbb{P}(\cdot)$ to be equivalent to an infinite horizon problem, $V_{f}(\cdot)$ can be chosen as follows:

$$
V_{f}(x, u):=\left\|\left[\begin{array}{l}
x-\bar{x} \\
u-\bar{u}
\end{array}\right]\right\|_{P}^{2}
$$

in which $(\bar{x}, \bar{u})$ are state and input steady-state targets, respectively, which satisfy:

$$
\begin{array}{r}
\bar{x}=A \bar{x}+B \bar{u}+\tilde{d}_{x} \\
\bar{y}=C \bar{x}+\tilde{d}_{y}
\end{array}
$$

with $\bar{u} \in \mathbb{U}$ and $\bar{y} \in \mathbb{Y}$. The matrix $P$ is the solution to the discrete-time algebraic Riccati equation for the following Linear Quadratic Regulation (LQR) matrices: $A_{P}:=\left[\begin{array}{cc}A & 0 \\ 0 & 0\end{array}\right]$, $B_{P}:=\left[\begin{array}{l}B \\ I\end{array}\right], Q_{P}:=\left[\begin{array}{ll}Q & 0 \\ 0 & R\end{array}\right], R_{P}:=R, M_{P}:=\left[\begin{array}{c}0 \\ -R\end{array}\right]$.

We observe that problem $\mathbb{P}(\cdot)$ is parametric in the current state $\tilde{x}$, the previously applied input $\tilde{u}$, and the current state/output disturbance pair $\left(\tilde{d}_{x}, \tilde{d}_{y}\right)$. Moreover, depending on the specific MPC formulation, the target tuple $(\bar{y}, \bar{x}, \bar{u})$ satisfying (7) can be regarded as a parameter of $\mathbb{P}(\cdot)$ when it is computed by a separate target calculation problem or as a decision variable of $\mathbb{P}(\cdot)$ in so-called single-layer MPC formulations.

\section{METHODS FOR OFFSET-FREE TRACKING}

In this Section, we review three different methods for offset-free MPC design within a coherent framework.

\section{A. Method 1: disturbance model and observer}

The first method to achieve offset-free tracking is to augment the nominal system dynamics (3) with additional integrating states, usually referred to as disturbances. The general augmented system can be written as follows [2]-[8]:

$$
\begin{aligned}
\hat{x}^{+} & =A \hat{x}+B u+B_{d} \hat{d} \\
\hat{d}^{+} & =\hat{d} \\
\hat{y} & =C \hat{x}+C_{d} \hat{d}
\end{aligned}
$$

in which $\hat{d} \in \mathbb{R}^{n_{d}}$ is the so-called disturbance. The evolution of the augmented state $\hat{\xi}:=\left[\begin{array}{l}\hat{x} \\ \hat{d}\end{array}\right]$ can be rewritten as

$$
\begin{gathered}
\hat{\xi}^{+}=A_{a} \hat{\xi}+B_{a} u \\
\hat{y}=C_{a} \hat{\xi}
\end{gathered}
$$

in which:

$$
A_{a}:=\left[\begin{array}{cc}
A & B_{d} \\
0 & I
\end{array}\right], \quad B_{a}:=\left[\begin{array}{c}
B \\
0
\end{array}\right], \quad C_{a}:=\left[\begin{array}{ll}
C & C_{d}
\end{array}\right]
$$

The following results are known [3], [5], and provide the basis for estimating the augmented state $\hat{\xi}$.

Proposition 3: The pair $\left(C_{a}, A_{a}\right)$ is detectable if and only if $(C, A)$ is detectable and

$$
\operatorname{rank}\left[\begin{array}{cc}
A-I & B_{d} \\
C & C_{d}
\end{array}\right]=n+n_{d}
$$

Proposition 4: There exist matrices $\left(B_{d}, C_{d}\right)$ such that condition (10) holds if and only if $n_{d} \leq p$.

Assuming that $\left(B_{d}, C_{d}\right)$ are chosen to satisfy (10), the augmented state is estimated at each time $k$ given the output measurement by means on the current observer for (8):

$$
\hat{\xi}(k)=A_{a} \hat{\xi}(k-1)+B_{a} u(k-1)+K_{a}\left(y(k)-\hat{y}^{*}(k)\right)
$$

in which

$$
K_{a}:=\left[\begin{array}{l}
K_{x} \\
K_{d}
\end{array}\right]
$$

is the augmented observer gain matrix, and $\hat{y}^{*}(k)$ is the predicted output given by

$$
\hat{y}^{*}(k):=C_{a}\left(A_{a} \hat{\xi}(k-1)+B_{a} u(k-1)\right)
$$


Finally, given the current augmented state $\hat{\xi}(k)=\left[\begin{array}{c}\hat{x}(k) \\ \hat{d}(k)\end{array}\right]$, the offset-free MPC algorithm based on Method 1 solves problem $\mathbb{P}(\cdot)$ in (6) using the following definitions:

$$
\tilde{x}:=\hat{x}(k), \quad \tilde{d}_{x}:=B_{d} \hat{d}(k), \quad \tilde{d}_{y}:=C_{d} \hat{d}(k)
$$

Remark 5: The augmented state formulation (8) is only necessary to obtain the pair $(\hat{x}(k), \hat{d}(k))$, and consequently to define $\left(\tilde{d}_{x}, \tilde{d}_{y}\right)$, but it is not used in $\mathbb{P}(\cdot)$.

\section{B. Method 2: state disturbance observer}

The second method, proposed by Tatjewski [9], [10], is not based on an augmented system.

At each time $k$, the current state is estimated by means of (4) and (5) for a given observer gain $K \in \mathbb{R}^{n \times p}$ chosen such that $(A-K C A)$ is Hurwitz. Given this estimate $\hat{x}(k)$, a state disturbance is consequently defined and computed as:

$$
\begin{aligned}
d_{x}(k) & :=\hat{x}(k)-(A \hat{x}(k-1)+B u(k-1)) \\
& =K(y(k)-C(A \hat{x}(k-1)+B u(k-1)))
\end{aligned}
$$

In addition an output correction term, which is necessary to ensure offset-free tracking, is defined as:

$$
d_{y}(k):=y(k)-C \hat{x}(k)
$$

Thus, the prediction model used, at time $k$, is given by:

$$
\begin{aligned}
\hat{x}^{+} & =A \hat{x}+B u+d_{x}(k) \\
\hat{y} & =C \hat{x}+d_{y}(k)
\end{aligned}
$$

Hence, the offset-free MPC algorithm based on Method 2 solves problem $\mathbb{P}(\cdot)$ in (6) using the following definitions:

$$
\tilde{x}:=\hat{x}(k), \quad \tilde{d}_{x}:=d_{x}(k), \quad \tilde{d}_{y}:=d_{y}(k)
$$

\section{Method 3: velocity form model}

The third method is based on the so-called velocity form model, which can be easily derived from (3) as:

$$
\begin{aligned}
\delta \hat{x}^{+} & =A \delta \hat{x}+B \delta u \\
\hat{y}^{+} & =\hat{y}+C A \delta \hat{x}+C B \delta u
\end{aligned}
$$

By subtracting the output target from both sides of the second equation, defining the augmented state $\hat{\xi}_{\delta}:=\left[\begin{array}{c}\delta \hat{x} \\ \hat{y}-\bar{y}\end{array}\right]$, and considering the model tracking error $\hat{e}:=\hat{y}-\bar{y}$ as the output, we can rewrite (19) as:

$$
\begin{aligned}
\hat{\xi}_{\delta}^{+} & =A_{\delta} \hat{\xi}_{\delta}+B_{\delta} \delta u \\
\hat{e} & =C_{\delta} \hat{\xi}_{\delta}
\end{aligned}
$$

in which

$$
A_{\delta}:=\left[\begin{array}{cc}
A & 0 \\
C A & I
\end{array}\right], \quad B_{\delta}:=\left[\begin{array}{c}
B \\
C B
\end{array}\right], \quad C_{\delta}:=\left[\begin{array}{ll}
0 & I
\end{array}\right]
$$

Let $\xi_{\delta}:=\left[\begin{array}{c}\delta x \\ y-\bar{y}\end{array}\right]$. From (1), we observe that the actual system evolution in velocity form is the following:

$$
\begin{aligned}
\xi_{\delta}^{+} & =A_{\delta} \xi_{\delta}+B_{\delta} \delta u+\left[\begin{array}{c}
\delta w \\
C \delta w+\delta v^{+}
\end{array}\right] \\
e & =C_{\delta} \xi_{\delta}
\end{aligned}
$$

in which we notice that $e:=y-\bar{y}$ is the actual tracking error. Therefore, from (20) and (22), it follows that the velocity form model is exact also in the presence of constant state and output disturbances, i.e. whenever $\delta w=0, \delta v=0$. For this reason, it does not need a disturbance model to compensate for constant nonzero disturbances.

The next result (proof, based on the Hautus Lemma, is omitted due to space limitations) provides the basis for estimating the augmented state of the velocity form $\hat{\xi}_{\delta}$.

Proposition 6: The pair $\left(C_{\delta}, A_{\delta}\right)$ is detectable if and only if $(C, A)$ is detectable.

Thus, under Assumption 1, the augmented state of the velocity form model (20) can be obtained from the measured tracking error by means of the current observer as follows:

$$
\hat{\xi}_{\delta}(k)=A_{\delta} \hat{\xi}_{\delta}(k-1)+B_{\delta} \delta u(k-1)+K_{\delta}\left(e(k)-\hat{e}^{*}(k)\right)
$$

in which

$$
K_{\delta}:=\left[\begin{array}{c}
K_{\delta x} \\
K_{e}
\end{array}\right], \quad \hat{e}^{*}(k):=C_{\delta}\left(A_{\delta} \hat{\xi}_{\delta}(k-1)+B_{\delta} \delta u(k-1)\right)
$$

Notice that $K_{\delta}$ should be chosen such that $\left(A_{\delta}-K_{\delta} C_{\delta} A_{\delta}\right)$ is Hurwitz. Most often, given that the second component of the velocity form state is a measurable quantity, namely the tracking error, it is customary to use a deadbeat observer for that state component, i.e. $K_{e}=I$, so that we obtain

$$
\hat{y}(k)-\bar{y}=e(k)=y(k)-\bar{y}
$$

From (25), it follows that the model output $\hat{y}(k)$ is implicitly realigned to the measured output $y(k)$ at each decision time.

Let $\tilde{\xi}_{\delta}$ denote the current augmented state estimate given by (23) and $\tilde{u}$ the previously applied input. When using the velocity form, the FHOCP (6) should be equivalently rewritten as follows, and denoted by $\mathbb{P}_{\delta}\left(\tilde{\xi}_{\delta}, \tilde{u}\right)$ :

$$
\begin{gathered}
\min _{\boldsymbol{\xi}, \boldsymbol{\delta} \boldsymbol{u}} \sum_{i=0}^{N-1} \ell\left(C_{\delta} \xi_{\delta}(i), \delta u(i)\right)+V_{f}^{\delta}\left(\xi_{\delta}(N)\right) \quad \text { s.t. } \\
\xi_{\delta}(0)=\tilde{\xi_{\delta}} \\
\xi_{\delta}(i+1)=A_{\delta} \xi_{\delta}(i)+B \delta u(i) \\
\tilde{u}+\sum_{j=0}^{i} \delta u(j) \in \mathbb{U}, \quad \delta u(i) \in \delta \mathbb{U} \\
C_{\delta} \xi_{\delta}(i)+\bar{y} \in \mathbb{Y}
\end{gathered}
$$

in which $\boldsymbol{\xi}=\left[\begin{array}{llll}\boldsymbol{\xi}(0) & \boldsymbol{\xi}(1) & \cdots & \boldsymbol{\xi}(N)\end{array}\right]$ and $\boldsymbol{\delta} \boldsymbol{u}=$ $\left[\begin{array}{llll}\delta u(0) & \delta u(1) & \cdots & \delta u(N-1)\end{array}\right]$ are the state and input sequences of the velocity form.

In order for $\mathbb{P}_{\delta}(\cdot)$ in (26) to be equivalent to an infinite horizon problem, the terminal cost function $V_{f}^{\delta}(\cdot)$ can be defined as: $V_{f}^{\delta}(\xi):=\|\xi\|_{P_{\delta}}^{2}$, in which $P_{\delta}$ is the solution to the discrete-time algebraic Riccati equation for the following LQR matrices: $A_{\delta}, B_{\delta}:=\left[\begin{array}{l}B \\ I\end{array}\right], Q_{\delta}:=\left[\begin{array}{ll}0 & 0 \\ 0 & Q\end{array}\right], R_{\delta}:=R$. Notice that existence of $P_{\delta}$ requires the pair $\left(A_{\delta}, B_{\delta}\right)$ to be stabilizable and $\left(Q_{\delta}^{1 / 2}, A_{\delta}\right)$ detectable. To this aim we have the following results (proofs, based on the Hautus Lemma, are omitted due to space limitations).

Proposition 7: The pair $\left(A_{\delta}, B_{\delta}\right)$ is stabilizable if and only if $(A, B)$ is stabilizable and condition (2) holds true. 
Proposition 8: The pair $\left(Q_{\delta}^{1 / 2}, A_{\delta}\right)$ is detectable if and only if $(C, A)$ is detectable and $Q$ is positive definite.

\section{MAIN RESUlTS}

The main results of this work are now presented. We show that Method 2 and Method 3 are particular cases of Method 1.

\section{A. Method 2 vs. Method 1}

Theorem 9: Method 2 described in Section III-B is a particular case of Method 1 described in Section III-A using:

$$
B_{d}=K, \quad C_{d}=I-C K, \quad K_{x}=K, \quad K_{d}=I
$$

and, for all $k \in \mathbb{Z}$ there holds $\hat{d}(k)=d_{y}(k)+C d_{x}(k)$.

Proof: Considering the disturbance model matrices defined in (27), from (9), (11) and (12), the evolution of the augmented state is given by:

$$
\begin{aligned}
{\left[\begin{array}{c}
\hat{x}(k) \\
\hat{d}(k)
\end{array}\right]=\left[\begin{array}{cc}
A & K \\
0 & I
\end{array}\right]\left[\begin{array}{c}
\hat{x}(k-1) \\
\hat{d}(k-1)
\end{array}\right]+} & {\left[\begin{array}{c}
B \\
0
\end{array}\right] u(k-1)+} \\
& {\left[\begin{array}{c}
K \\
I
\end{array}\right]\left(y(k)-\hat{y}^{*}(k)\right) }
\end{aligned}
$$

in which, from (13), the predicted output $\hat{y}^{*}(k)$ is given by:

$$
\begin{array}{r}
\hat{y}^{*}(k)=\left[\begin{array}{cc}
C & I-C K
\end{array}\right]\left(\left[\begin{array}{cc}
A & K \\
0 & I
\end{array}\right]\left[\begin{array}{c}
\hat{x}(k-1) \\
\hat{d}(k-1)
\end{array}\right]+\left[\begin{array}{l}
B \\
0
\end{array}\right] u(k-1)\right) \\
=C A \hat{x}(k-1)+C B u(k-1)+\hat{d}(k-1)
\end{array}
$$

From (28) and (29), after simplification, it follows that:

$$
\begin{aligned}
\hat{x}(k)= & A \hat{x}(k-1)+B u(k-1) \\
& \quad+K(y(k)-C(A \hat{x}(k-1)+B u(k-1)) \\
= & A \hat{x}(k-1)+B u(k-1)+d_{x}(k) \\
\hat{d}(k)= & y(k)-C(A \hat{x}(k-1)+B u(k-1)) \\
= & d_{y}(k)+C d_{x}(k)
\end{aligned}
$$

which, by inspection of (15)-(16), completes the proof.

We next show that the augmented system (implicitly) used by Method 2 is detectable, and that the associated augmented observer is asymptotically stable.

Proposition 10: Consider an augmented system (8)-(9) with matrices $B_{d}:=K, C_{d}:=I-C K$, and $K$ chosen such that $(A-K C A)$ is Hurwitz. Then, the detectability condition of the augmented system (10) holds true.

Proof: To show that (10) holds, consider the system:

$$
\left[\begin{array}{cc}
A-I & K \\
C & I-C K
\end{array}\right]\left[\begin{array}{l}
x \\
y
\end{array}\right]=\left[\begin{array}{l}
0 \\
0
\end{array}\right]
$$

or equivalently:

$$
(A-I) x+K y=0, \quad C x+(I-C K) y=0
$$

Rearranging (31) we obtain:

$$
(A-I) x+K y=0, \quad y+C A x=0
$$

and combining (32) we obtain:

$$
(A-K C A-I) x=0 \Rightarrow x=0
$$

where the last step comes from the fact that $(A-K C A)$ is Hurwitz, and hence $(A-K C A-I)$ is invertible. Finally, $y+$ $C A x=0$ and $x=0$ imply $y=0$. Thus, the unique solution to (30) is $\left[\begin{array}{l}x \\ y\end{array}\right]=\left[\begin{array}{l}0 \\ 0\end{array}\right]$, which completes the proof.

Proposition 11: Consider an augmented system observer (8), (9), (11), (12) with matrices $B_{d}:=K, C_{d}:=I-C K$, $K_{x}:=K, K_{d}:=I$, and $K$ chosen such that $(A-K C A)$ is Hurwitz. Then, the associated augmented observer matrix $\left(A_{a}-K_{a} C_{a} A_{a}\right)$ is Hurwitz.

Proof: We can write:

$$
\begin{array}{r}
A_{a}-K_{a} C_{a} A_{a}=\left[\begin{array}{cc}
A & K \\
0 & I
\end{array}\right]-\left[\begin{array}{c}
K \\
I
\end{array}\right]\left[\begin{array}{ll}
C & I-C K
\end{array}\right]\left[\begin{array}{cc}
A & K \\
0 & I
\end{array}\right] \\
=\left[\begin{array}{cc}
A & K \\
0 & I
\end{array}\right]-\left[\begin{array}{cc}
K C A & K \\
C A & I
\end{array}\right]=\left[\begin{array}{cc}
A-K C A & 0 \\
-C A & 0
\end{array}\right]
\end{array}
$$

Thus, the matrix $\left(A_{a}-K_{a} C_{a} A_{a}\right)$ has the same eigenvalues of $(A-K C A)$ and $p$ zero eigenvalues. Since by assumption $(A-K C A)$ is Hurwitz, the claim is proved.

Remark 12: From (33) we notice that the augmented system observer (implicitly) used in Method 2, is deadbeat with respect to the mixed state/output disturbance, $\hat{d}=d_{y}+C d_{x}$, and does not alter the closed-loop dynamics of the state estimate error still governed by $(A-K C A)$.

\section{B. Method 3 vs. Method 1}

Proposition 13: Consider the velocity form observer (23)(24) with $K_{e}=I$. The associated matrix $\left(A_{\delta}-K_{\delta} C_{\delta} A_{\delta}\right)$ is Hurwitz if and only if $K_{\delta x}$ is chosen such that $\left(A-K_{\delta x} C A\right)$ is Hurwitz.

Proof: We can write:

$$
\begin{aligned}
\left(A_{\delta}-K_{\delta} C_{\delta} A_{\delta}\right) & =\left[\begin{array}{cc}
A & 0 \\
C A & I
\end{array}\right]-\left[\begin{array}{c}
K_{\delta x} \\
I
\end{array}\right]\left[\begin{array}{ll}
0 & I
\end{array}\right]\left[\begin{array}{cc}
A & 0 \\
C A & I
\end{array}\right] \\
& =\left[\begin{array}{cc}
A & 0 \\
C A & I
\end{array}\right]-\left[\begin{array}{c}
K_{\delta x} \\
I
\end{array}\right]\left[\begin{array}{ll}
C A & I
\end{array}\right] \\
& =\left[\begin{array}{cc}
A & 0 \\
C A & I
\end{array}\right]-\left[\begin{array}{cc}
K_{\delta x} C A & K_{\delta x} \\
C A & I
\end{array}\right]
\end{aligned}
$$

and finally

$$
\left(A_{\delta}-K_{\delta} C_{\delta} A_{\delta}\right)=\left[\begin{array}{cc}
A-K_{\delta x} C A & -K_{\delta x} \\
0 & 0
\end{array}\right]
$$

which completes the proof.

Remark 14: From (34) we notice that $\left(A_{\delta}-K_{\delta} C_{\delta} A_{\delta}\right)$ has $p$ eigenvalues at the origin, which are associated with the deadbeat estimation of the tracking error, in accordance with (25).

Theorem 15: Method 3 described in Section III-C, with $K_{e}=I$, is a particular case of Method 1 described in Section III-A using:

$$
B_{d}=K_{\delta x}, \quad C_{d}=I-C K_{\delta x}, \quad K_{x}=K_{\delta x}, \quad K_{d}=I
$$

Proof: Consider the velocity form observer (23)-(24) with $K_{e}=I$. By expanding and rearranging the various terms in (23)-(24), and recalling (21) and (34), we obtain:

$$
\begin{aligned}
\delta \hat{x}(k)= & \left(A-K_{\delta x} C A\right) \delta \hat{x}(k-1)+ \\
& \left(B-K_{\delta x} C B\right) \delta u(k-1)+K_{\delta x}(e(k)-\hat{e}(k-1)) \\
\hat{e}(k)= & e(k)
\end{aligned}
$$


which can also be rewritten as:

$$
\begin{aligned}
\delta \hat{x}(k)= & \left(A-K_{\delta x} C A\right) \delta \hat{x}(k-1)+ \\
& \left(B-K_{\delta x} C B\right) \delta u(k-1)+K_{\delta x}(e(k)-e(k-1))
\end{aligned}
$$

Now consider the evolution of the augmented system of Method 1 with matrices given in (35), which can be written:

$$
\begin{aligned}
\hat{x}(k)=A \hat{x}(k-1)+B u(k-1) & \\
& +K_{\delta x}(y(k)-C(A \hat{x}(k-1)+B u(k-1)))
\end{aligned}
$$

By rewriting (37) at time $k-1$ and taking the difference of both sides, we obtain:

$$
\begin{array}{r}
\delta \hat{x}(k)=A \delta \hat{x}(k-1)+B \delta u(k-1)+K_{\delta x}(y(k)-y(k-1)) \\
\left.-K_{\delta x} C(A \delta \hat{x}(k-1)+B \delta u(k-1))\right)
\end{array}
$$

Since there holds:

$$
\begin{aligned}
& y(k)-y(k-1)=(y(k)-\bar{y})-(y(k-1)-\bar{y}) \\
&=e(k)-e(k-1)
\end{aligned}
$$

we can rewrite (38) as

$$
\begin{aligned}
& \delta \hat{x}(k)=\left(A-K_{\delta x} C A\right) \delta \hat{x}(k-1)+ \\
& \left(B-K_{\delta x} C B\right) \delta u(k-1)+K_{\delta x}(e(k)-e(k-1))
\end{aligned}
$$

The proof is completed by comparing (40) with (36).

Remark 16: By comparing Thm. 9 and Thm. 15 we easily see that Method 2 and Method 3 (with $K_{e}=I$ ) are also equivalent to each other if $K=K_{\delta x}$.

\section{Tuning}

One aspect that is not straightforward in the design of offset-free MPC systems is the choice of various matrices that appear in the algorithm. This difficulty is common to all methods, in which at least an observer gain needs to be defined, but it is clear Method 1 requires the choice of the disturbance model matrices $\left(B_{d}, C_{d}\right)$ and of the augmented observer gain matrices $\left(K_{x}, K_{d}\right)$. An important aspect to remark is that, in general any choice of $\left(B_{d}, C_{d}\right)$ respecting (10) is equivalent because there exist observer gain matrices $\left(K_{x}, K_{d}\right)$ which make the augmented system defined by the tuple $\left(A_{a}, B_{a}, C_{a}, K_{a}\right)$ algebraically equivalent to any desired augmented system based on the same nominal model matrices $(A, B, C)$ but different $\left(B_{d}, C_{d}\right)$ (see [15], [16] for details). Therefore, strictly speaking, only $\left(K_{x}, K_{d}\right)$ need to be chosen.

The equivalence results of Thm. 9 and Thm. 15 suggest a simple and unified approach for tuning $\left(B_{d}, C_{d}, K_{x}, K_{d}\right)$ in Method 1 (or $\left(K_{\delta x}, K_{e}\right)$ in Method 3) which follows the approach of Method 2. First, choose the observer gain $K$ such that the non-augmented system characteristic matrix $(A-K C A)$ has desired properties, e.g. assigned eigenvalues. Then, for Method 1 , use (27) to define $\left(B_{d}, C_{d}, K_{x}, K_{d}\right)$. For Method 3 instead, use $K_{\delta x}=K$ and $K_{e}=I$. Clearly, this simple tuning may be sensitive to output noise because it places $p$ observer poles at the origin. This aspect is highlighted by an illustrative example in the next section.

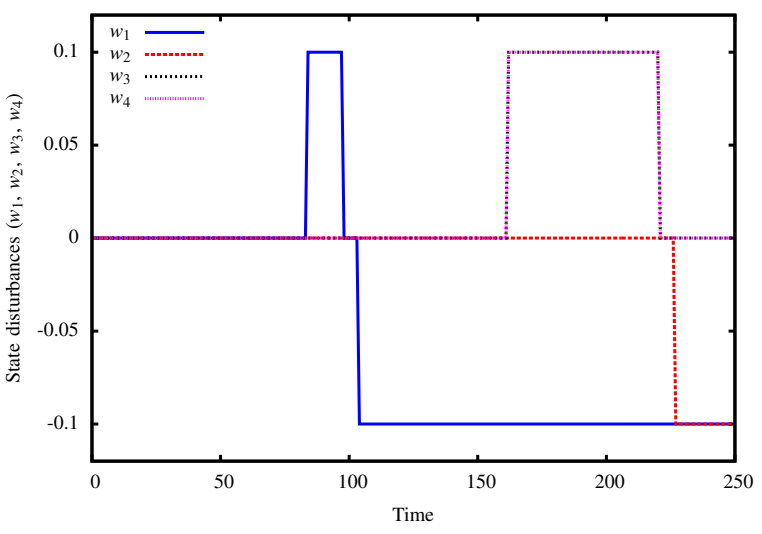

Fig. 1. Time varying state disturbances.

\section{ILLUSTRATIVE EXAMPLE}

As illustrative example, we consider the 2 input, 2 output system defined by the matrices [10]:

$$
\begin{aligned}
A=\left[\begin{array}{cccc}
0.958 & 0 & 0 & 0 \\
0 & 0.9418 & 0 & 0 \\
0 & 0 & 0.9048 & 0 \\
0 & 0 & 0 & 0.9277
\end{array}\right], \quad B=\left[\begin{array}{cc}
0.25 & 0 \\
0.25 & 0 \\
0 & 0.5 \\
0 & 0.5
\end{array}\right], \\
C=\left[\begin{array}{cccc}
0.1678 & 0 & 0.9516 & 0 \\
0 & 0.2329 & 0 & 0.289
\end{array}\right]
\end{aligned}
$$

Inputs are assumed to be constrained, $\|u\|_{\infty}=2$, and the output is required to track a piece wise constant target despite the presence of (unmeasured) time varying disturbances on the state dynamics, $w(k)$ for all $k \in \mathbb{Z}$, as depicted in Fig. 1. The output measurement is affected by normally distributed random noise, $v(k)$ for all $k \in \mathbb{Z}$. We compare three MPC algorithms:

- MPC-0 is a standard (non offset-free) algorithm, i.e. it uses $\tilde{d}_{x}=0, \tilde{d}_{y}=0$. The state observer gain $K$ in (4) is computed such that the observer poles are at $(0.60,0.61,0.62,0.63)$.

- MPC-1 uses Method 2 with the same observer gain $K$ as MPC-0.

- MPC-2 uses Method 1 with disturbance model matrices chosen such that $\left[\begin{array}{l}B_{d} \\ C_{d}\end{array}\right]$ is an orthonormal basis of null space of $\left[\begin{array}{c}A-I \\ C\end{array}\right]^{\prime}$, and the augmented observer gain is $K_{a}$ is computed such that the observer poles are at $(0.60,0.61,0.62,0.63,0.50,0.50)$.

For all algorithms we use: $N=100, Q=I, R=I$.

Given the results of this paper, MPC-1 based on Method 2 is also equivalent to using Method 1 with $B_{d}=K, C_{d}=$ $I-C K, K_{x}=K, K_{d}=I$ or Method 3 with $K_{\delta x}=K$ and $K_{e}=I$. Moreover, the augmented observer poles of MPC-1 are inherently placed at $(0.60,0.61,0.62,0.63,0,0)$.

Comparative results are reported in Fig. 2. As expected MPC-0 is not able to track the desired target without offset, whereas MPC-1 and MPC-2 eliminate permanent offset. The behavior of MPC-1 and MPC-2 is similar in terms of outputs, but the input generated by MPC-1 appears less smooth than that of MPC-2. This behavior occurs due to the fact that MPC-1 has two observer poles at the origin, whereas in MPC-2 the corresponding poles are placed at 0.5 , and hence 

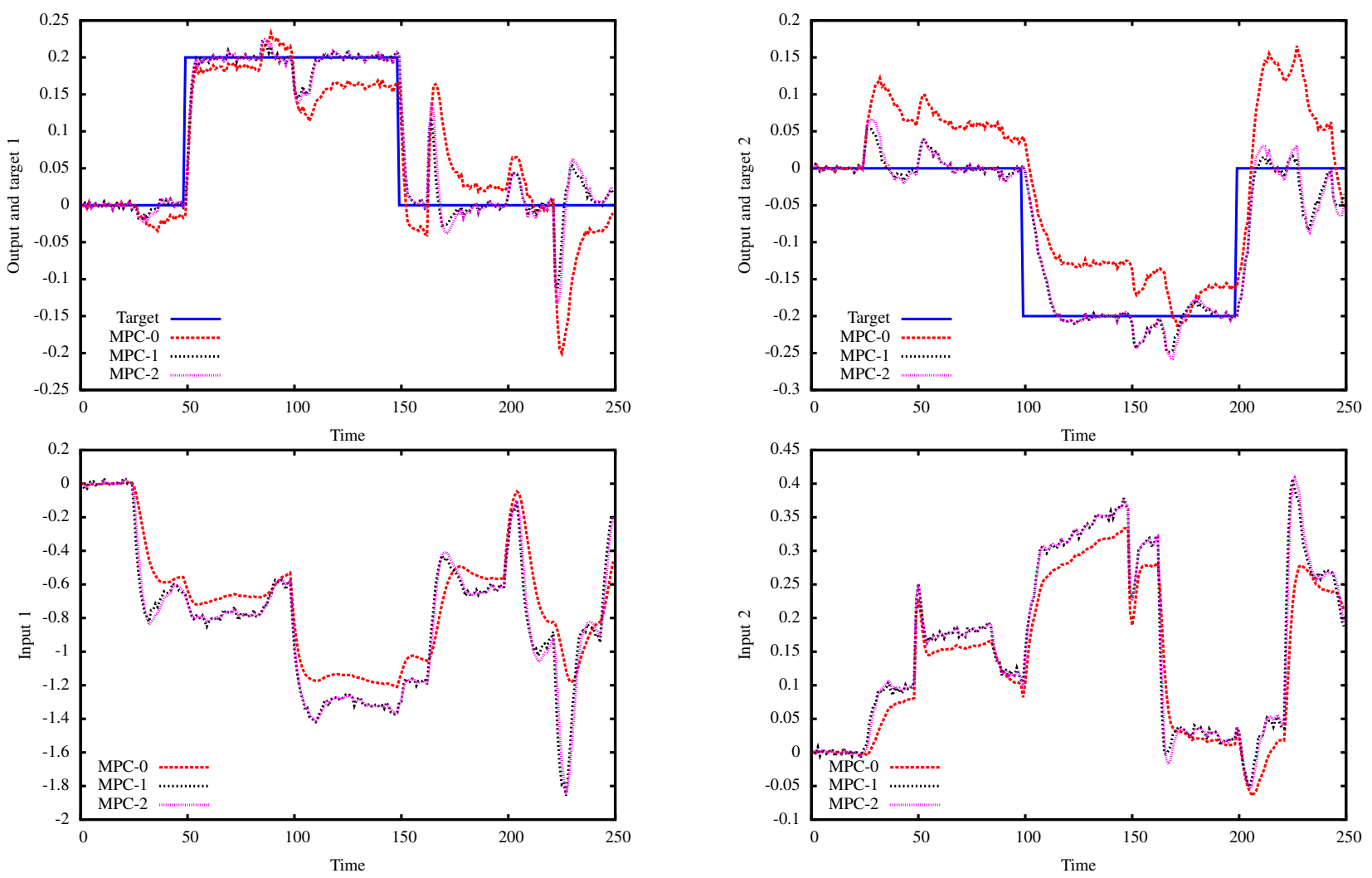

Fig. 2. Closed-loop outputs (top) and inputs (bottom) using MPC-0, MPC-1 and MPC-2. Output target is also shown in top plots.

are less sensitive to measurement noise. The closed-loop cost function, $J_{C L}=\sum_{k}\|y(k)-\bar{y}(k)\|_{Q}^{2}+\|u(k)-u(k-1)\|_{R}^{2}$, is equal to 1.356 for MPC-0, 1.017 for MPC-1 and 0.934 for MPC-2. Thus, compared to MPC-2, MPC-0 is $45.2 \%$ suboptimal and MPC-1 is $8.84 \%$ suboptimal.

\section{CONCLUSIONS}

Offset-free MPC algorithms are usually designed by augmenting the nominal system with a disturbance model, and estimating the augmented state with an observer. This paper showed that two known (and supposedly alternative) offset-free MPC formulations, namely the state disturbance observer approach [10] and the velocity form approach, are particular cases of the disturbance model formulation. It is therefore no longer appropriate to consider them as alternative formulations, but simply as particular choices of the general approach. Hence, the debate about which formulation is better, simpler or more generally applicable should be reconsidered.

\section{REFERENCES}

[1] D. M. Prett and C. E. Garcia, Fundamental Process Control. Butterworth Publishers, Boston, 1988.

[2] J. B. Rawlings, "Tutorial overview of model predictive control," IEEE Control System Magazine, vol. 20, pp. 38-52, June 2000.

[3] K. R. Muske and T. A. Badgwell, "Disturbance modeling for offsetfree linear model predictive control," J. Proc. Cont., vol. 12, pp. 617632, 2002.

[4] G. Pannocchia, "Robust disturbance modeling for model predictive control with application to multivariable ill-conditioned processes," $J$. Proc. Cont., vol. 13, pp. 693-701, 2003.
[5] G. Pannocchia and J. B. Rawlings, "Disturbance models for offset-free model predictive control," AIChE J., vol. 49, pp. 426-437, 2003.

[6] G. Pannocchia and A. Bemporad, "Combined design of disturbance model and observer for offset-free model predictive control," IEEE Trans. Auto. Contr., vol. 52, no. 6, pp. 1048-1053, 2007.

[7] U. Maeder, F. Borrelli, and M. Morari, "Linear offset-free model predictive control," Automatica, vol. 45, no. 10, pp. 2214-2222, 2009.

[8] U. Maeder and M. Morari, "Offset-free reference tracking with model predictive control," Automatica, vol. 46, no. 9, pp. 1469-1476, 2010.

[9] P. Tatjewski, Advanced Control of Industrial Processes. Springer Verlag, 2007.

[10] _ "Disturbance modeling and state estimation forr offset-free predictive control with state-space process models," Int. J. Appl. Math. Comput. Sci., vol. 24, no. 2, pp. 313-323, 2014.

[11] G. Pannocchia and J. B. Rawlings, "The velocity algorithm LQR: a survey," TWMCC, Department of Chemical Engineering, University of Wisconsin-Madison, Tech. Rep. 2001-01, May 2001, uRL: http://www.che.wisc.edu/jbr-group/tech-reports/twmcc-2001-01.pdf.

[12] A. H. González, E. J. Adam, and J. L. Marchetti, "Conditions for offset elimination in state space receding horizon controllers: A tutorial analysis," Chem. Engng. and Proc.: Process Intensification, vol. 47, no. 12, pp. 2184-2194, 2008.

[13] G. Betti, M. Farina, and R. Scattolini, "An MPC algorithm for offsetfree tracking of constant reference signals," in Proceedings of 51st IEEE Conference on Decision and Control, Maui, Hawaii, USA, 2012, pp. 5182-5187.

[14] —, "A robust MPC algorithm for offset-free tracking of constant reference signals," IEEE Trans. Auto. Contr., vol. 58, no. 9, pp. 2394 2400, 2013.

[15] G. Pannocchia, A. De Luca, and M. Bottai, "Prediction error based performance monitoring, degradation diagnosis and remedies in offsetfree MPC: Theory and applications," Asian J. of Control, vol. 16, no. 4, pp. 995-1005, 2014.

[16] M. R. Rajamani, J. B. Rawlings, and S. J. Qin, "Achieving state estimation equivalence for misassigned disturbances in offset-free model predictive control," AIChE J., vol. 55, pp. 396-407, 2009. 